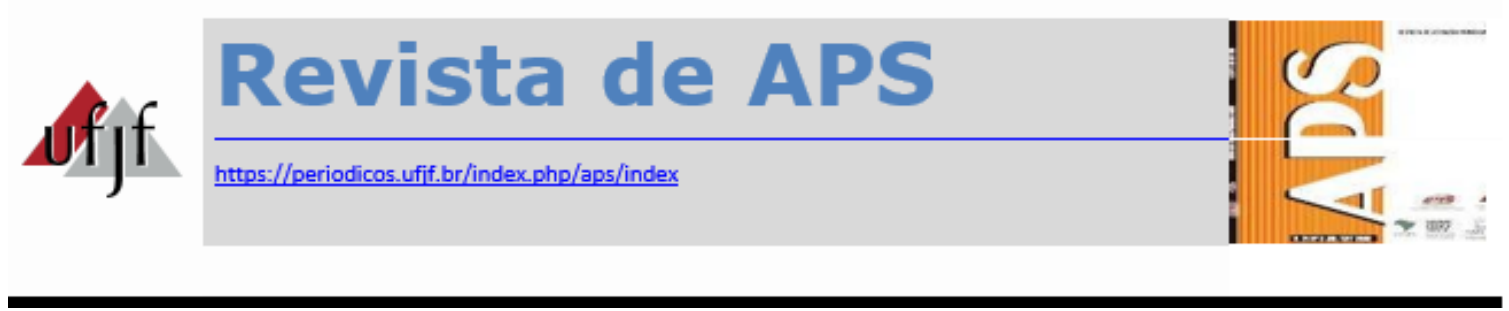

\title{
Avaliação das estratégias de educação em saúde com adolescentes
}

\author{
Evaluation of health education strategies with adolescents
}

\author{
Rubênia Paulo da Silva ${ }^{1}$, Rafaela Carolini de Oliveira Távora ${ }^{2}$, José Adaílton da Silva ${ }^{3}$, Mayara \\ Silva Fernandes do Rêgo ${ }^{4}$
}

\section{RESUMO}

Introdução: considerando a importância e a relevância da educação em saúde com adolescentes, é essencial o uso de estratégias cada vez mais acolhedoras, dinâmicas, integradas e subjetivas. Assim, objetivou-se avaliar as estratégias de educação em saúde utilizadas com adolescentes, a partir das publicações existentes em revistas científicas. Desenvolvimento: trata-se de uma pesquisa descritiva, qualitativa, do tipo revisão integrativa. Foram critérios de inclusão: títulos em português, texto completo disponível gratuitamente, artigos e publicações nos últimos cinco anos (2012, 2013, 2014, 2015 e 2016). Foram excluídos os artigos repetidos ou em formato de revisão de literatura ou que não responderam à questão-problema pesquisada. Após a aplicação dos critérios de inclusão e exclusão, restaram 26 artigos, que foram lidos na íntegra e analisados na presente pesquisa. A região Nordeste destacou-se com dez publicações e a revista APS Atenção Primária à Saúde possui o maior número de publicações. A maioria dos artigos utilizou a abordagem qualitativa. As oficinas educativas foram relatadas em 12 pesquisas, e abordou-se a temática saúde sexual e reprodutiva na maioria das ações. As escolas foram os cenários de maior escolha pelos pesquisadores. Em média, $69 \%$ dos artigos não utilizaram base metodológica para avaliar a efetividade das intervenções. Evidenciaram-se, ainda, limitações das publicações quanto às conclusões e avaliações das estratégias utilizadas. Conclusão: reforça-se a importância da pesquisa na área profissional, o que garante tanto a importância da

\footnotetext{
${ }^{1}$ Enfermeira. Universidade Federal do Rio Grande do Norte (UFRN).

${ }^{2}$ Doutora em Enfermagem pela Universidade Federal do Ceará (UFC). Docente da Faculdade de Ciências da Saúde do Trairi/Universidade Federal do Rio Grande do Norte (Facisa/UFRN). E-mail: profenfrafaela@gmail.com.

${ }^{3}$ Mestrado em Saúde da Família pela Universidade Federal do Rio Grande do Norte (UFRN).

${ }^{4}$ Docente do curso de graduação em Enfermagem. Docente da Faculdade de Ciências da Saúde do Trairi/Universidade Federal do Rio Grande do Norte (Facisa/UFRN).
} 
busca de metodologias quanto da avaliação da estratégia usada, para melhor aplicabilidade, de forma dinâmica, afável e pessoal.

PALAVRAS-CHAVE: Adolescente; Educação; Saúde.

\begin{abstract}
Introduction: considering the importance and relevance of health education with adolescents, it is essential to use strategies that are increasingly welcoming, dynamic, integrated and subjective. Thus, the objective was to evaluate strategies of health education used with adolescents, based on existing publications in scientific journals. Development: this is a descriptive, and qualitative research of the integrative review type. Inclusion criteria were the following: titles in Portuguese, full text freely available, articles and publications from the last five years $(2012,2013,2014,2015$ and 2016). Articles that were repeated or in a literature review format or that did not respond to the problem question researched were excluded. After applying the inclusion and exclusion criteria, there were 26 articles, which were fully read and analyzed in the present research. The Northeast region was prominent with 10 publications and the Primary Health Care magazine had the largest number of publications, with most articles using a qualitative approach. Educational workshops were reported in 12 surveys and sexual and reproductive health issues were addressed in most actions. Schools were the most chosen (or "frequent choice") scenarios for articles. On average, 69\% of the articles did not use a methodological basis to evaluate the effectiveness of the interventions. Limitations of the publications regarding the conclusions and evaluations of the strategies used superficially were also evidenced. Conclusion: it reinforces the importance of the research in the professional area, ensuring both the necessity of searching for methodologies, and evaluating the strategy used, for a better applicability in a dynamic, affable and personal way.
\end{abstract}

KEYWORDS: Adolescent; Education; Health.

\title{
INTRODUÇÃO
}

Nos últimos 20 anos, a atenção à saúde do adolescente ganhou significativa importância em diversos países e, até mesmo, em instituições internacionais voltadas à promoção de pesquisas. Isso comprova que a educação em saúde promotora de um modo de vida saudável para esse público é decisiva, não apenas para o jovem, como também para as gerações posteriores. ${ }^{1}$ Abordar tal público é um desafio, pois a adolescência possui marcantes transformações multidimensionais, envolvendo um contexto biológico, psicológico e sociocultural. A adolescência consiste em um período de mudanças e conflitos que, muitas vezes, não são acompanhados de maneira satisfatória pela família ou por profissionais envolvidos no cuidado. ${ }^{2}$ 
Cada experiência pela qual o jovem passa pode afetar seu futuro, por meio da influência de amigos e pessoas que o cercam, influenciando-o em suas práticas e podendo gerar mudanças de comportamento e atitudes.

A adolescência vai apresentando para o ser humano uma identificação pessoal, sexual e laboral, permitindo-o desempenhar diversas atribuições na sociedade. As concepções elaboradas nesse tempo expressam-se em sua maneira de ser no mundo, em suas funções sociais. ${ }^{1}$

Essa é uma época de profundas mudanças e transformações que, muitas vezes, configuram-se como vulnerabilidades, devido à falta do conhecimento, que podem resultar em gravidez não planejada, abortos, contaminação por doenças sexualmente transmissíveis, uso de álcool e outras drogas, violências físicas, psicológicas, familiares e diversos outros tipos de agravos. ${ }^{1}$ Desse modo, é necessário empoderar o adolescente para que ele possa se proteger dos agravos, riscos e vulnerabilidades aos quais pode estar exposto, na busca por um caminho que utilize melhor seu potencial.

A execução de metodologias educativas com adolescentes, direcionadas para a formação de competências, mostra-se de grande importância. A educação em saúde oportuniza ao adolescente evitar condutas de risco para sua saúde e seu desenvolvimento. Assim, é possível instigar a autonomia do jovem no combate à vulnerabilidade, tendo em vista os riscos a que essa faixa etária está exposta. ${ }^{3}$

O uso de estratégias ou metodologias ativas na educação em saúde contribui para o autoconhecimento e o aumento de capacidades dos adolescentes, além de estimular a sensibilidade, a inteligência e a compreensão acerca de diversos assuntos, tornando o adolescente multiplicador de conhecimentos. ${ }^{4}$ É imprescindível para o cuidado em saúde dos adolescentes utilizar estratégias integradas e intersetoriais para proporcionar saúde e prevenir doenças e agravos. ${ }^{5}$ Contudo, a abordagem e o trabalho de educação em saúde dos profissionais com esse público têm sido dificultados, pela não obediência aos direitos do adolescente. Acrescenta-se que sociedade e Estado não estão aptos a cuidar adequadamente desse grupo. ${ }^{1}$

Para os adolescentes, as estratégias participativas são as metodologias de educação em saúde que suprem da melhor forma as perspectivas em abrangência de atividades. Desse modo, o debate em grupo beneficia o diálogo, um método ativo que se amplia entre sujeitos, arrastando todos à demonstração franca e aberta dos 
pensamentos. $^{2}$

O desenvolvimento de educação em saúde surge oportunizando-se dos diversos ambientes com diferentes estratégias. Salientam-se as rodas de terapia, o acolhimento individual, as ações na atenção básica e a realização de oficinas, as ações com o Núcleo de Apoio a Saúde da Família (Nasf), além de Bombeiros, Polícia e Conselho Local de Saúde, que são organizados na tentativa de minimização da vulnerabilidade nos adolescentes. Todavia, a relação dos profissionais com esses serviços é, por vezes, breve; e as atuações intersetoriais são fracas. ${ }^{6}$

É importante ressaltar a variedade de locais em que as metodologias podem ser aplicadas, como escolas, igrejas, praças, centros urbanos, clínicas, setores hospitalares, nas quais os adolescentes encontram-se internados, em tratamento e outros. Entretanto, são diversas as dificuldades relatadas para a realização de atividades com os adolescentes: a baixa receptividade de alguns adolescentes e seus pais; os "tabus" advindos das gerações menos escolarizadas tanto dos familiares quanto dos educadores; o desinteresse dos profissionais em ir ao encontro desse grupo populacional; o excesso de demanda de atendimentos; a falta de políticas públicas e de capacitação para a equipe; as limitações do espaço físico para atividades; e as dificuldades de inserção nos ambientes em que esses adolescentes estão, como clínicas, escolas e programas instaurados na comunidade. ${ }^{6}$

A promoção da saúde na adolescência é um desafio que inclui a intersetorialidade, a capacitação e a articulação profissional para os que trabalham com este grupo populacional. É importante também afirmar o papel da gestão em saúde, que deve incentivar políticas que promovam a saúde do adolescente.

Nesse contexto, é importante não só desenvolver estratégias de educação em saúde, mas avaliar a efetividade das ações, como forma do desenvolvimento de uma consciência crítico-reflexiva quanto às estratégias aplicadas. ${ }^{7}$ Considerando a importância e a relevância da educação em saúde com adolescentes, é essencial o uso de estratégias cada vez mais acolhedoras, dinâmicas, integradas e subjetivas. Assim, o presente trabalho objetiva avaliar as estratégias de educação em saúde utilizadas com adolescentes. 


\section{DESENVOLVIMENTO}

A metodologia empregada na presente pesquisa foi descritiva e qualitativa, sendo uma revisão de literatura do tipo revisão integrativa. A revisão integrativa da literatura consiste na elaboração de uma vasta apreciação da bibliografia, com a intenção de contribuir para discussões sobre metodologias e resultados de pesquisas, também como para cogitações sobre futuros estudos a serem realizados. ${ }^{8}$

Para a construção da revisão de literatura, fez-se necessário seguir etapas para a composição do estudo: identificação da questão de pesquisa, estabelecimento de critérios da amostragem, identificação, avaliação, interpretação dos resultados e apresentação do estudo. ${ }^{8}$ A fim de nortear a pesquisa, estabeleceu-se a seguinte questão: quais as estratégias de educação em saúde utilizadas com adolescentes? As palavras-chave foram escolhidas pelos Descritores em Ciências da Saúde presentes na BVS (DeCS): adolescente, educação e saúde.

As publicações foram captadas no banco de dados Biblioteca Virtual em Saúde (BVS), que está composta de bases de dados bibliográficas produzidas pela Rede BVS, como Literatura Latino-americana e do Caribe em Ciências da Saúde (Lilacs), Medical Literature Analysis and Retrieval System Online (Medline) e Scientific Electronic Library Online (Scielo). A pesquisa foi realizada no dia 12 de julho de 2016. Utilizaram-se os descritores "adolescente", "educação" e "saúde"; com o operador booleano "and".

Os critérios de inclusão foram: estudos em português, texto completo disponível e documento do tipo "artigo", publicados nos últimos cinco anos, por serem pesquisas mais recentes. Foram excluídos os artigos repetidos ou em formato de revisão de literatura ou que não respondessem à questão pesquisada. Os títulos e resumos foram avaliados; e as pesquisas que atenderam aos critérios previamente instituídos, selecionadas e lidas na íntegra. Os artigos selecionados são apresentados na próxima página no Quadro 1. 
Quadro 1 - Descrição dos artigos conforme título, autor, ano, equipe pesquisadora, local, periódico, tipo de estudo e local da intervenção

\begin{tabular}{|c|c|c|c|}
\hline Título & $\begin{array}{l}\text { Autor/ano/equipe } \\
\text { pesquisadora/local }\end{array}$ & $\begin{array}{l}\text { Periódico/tipo de } \\
\text { estudo }\end{array}$ & $\begin{array}{c}\text { Local } \\
\text { da } \\
\text { interv. }\end{array}$ \\
\hline $\begin{array}{l}\text { Educação em saúde } \\
\text { com adolescentes: } \\
\text { compartilhando } \\
\text { Vivências e reflexões }\end{array}$ & $\begin{array}{l}\text { Coelho MMF, Raimundo } \\
\text { AMT, Karla CLM et } \\
\text { al./2012/enfermeiros e } \\
\text { acadêmicos./ CE }\end{array}$ & $\begin{array}{c}\text { Cienc Cuid } \\
\text { Saúde/qualitativo - } \\
\text { relato de experiência }\end{array}$ & $E$ \\
\hline $\begin{array}{l}\text { Oficinas educativas } \\
\text { como estratégia de } \\
\text { promoção da saúde } \\
\text { auditiva do } \\
\text { adolescente: estudo } \\
\text { exploratório }{ }^{10} \\
\end{array}$ & $\begin{array}{c}\text { Lacerda ABM, Soares } \\
\text { VMN, Gonçalves CGO, } \\
\text { Lopes FC, Testoni } \\
\text { R/fonoaudiólogo e } \\
\text { enfermeiro/2013/ PR }\end{array}$ & $\begin{array}{c}\text { ACR/qualitativo - } \\
\text { pesquisa - intervenção }\end{array}$ & EM / EP \\
\hline $\begin{array}{l}\text { Sentidos sobre } \\
\text { "sexualidade" e } \\
\text { "drogas" entre } \\
\text { adolescentes no } \\
\text { contexto escolar }{ }^{11}\end{array}$ & $\begin{array}{l}\text { Barros JPP, Rodrigues VF/ } \\
\text { psicólogos/2013/ CE }\end{array}$ & $\begin{array}{c}\text { EPP/intervenção - } \\
\text { qualitativa }\end{array}$ & $\mathrm{EP} / \mathrm{EF}$ \\
\hline $\begin{array}{l}\text { Intervenção educativa } \\
\text { utilizando a atividade } \\
\text { de vida respiração com } \\
\text { adolescentes }^{12}\end{array}$ & $\begin{array}{c}\text { Bezerra EP, Sousa LBM, } \\
\text { Alves } \\
\text { DS/enfermeiros/2014/CE }\end{array}$ & $\begin{array}{l}\text { Eanre/qualitativa - } \\
\text { pesquisa-ação }\end{array}$ & EJA / E \\
\hline $\begin{array}{c}\text { Educação sexual na } \\
\text { escola a partir da } \\
\text { Psicologia histórico- } \\
\text { cultural }^{13}\end{array}$ & $\begin{array}{l}\text { Bortolozzi ACM, Eidt NM, } \\
\text { Terra BM, Maia } \\
\text { GL/psicólogos/ 2012/ SP. }\end{array}$ & $\begin{array}{c}\text { Psicologia em } \\
\text { estudo/interven ção - } \\
\text { qualitativa. }\end{array}$ & EMp \\
\hline $\begin{array}{l}\text { Cultura masculina e } \\
\text { religiosidade na } \\
\text { prevenção de } \\
\text { DSTs/HIV/ aids em } \\
\text { adolescentes }^{14}\end{array}$ & $\begin{array}{c}\text { Ferreira AGN Kelanne LS, } \\
\text { Pedro } \\
\text { RMS./2012/enfermeiros e } \\
\text { acadêmicos de } \\
\text { Enfermagem/ CE }\end{array}$ & $\begin{array}{l}\text { Reme - Rev Min } \\
\text { Enferm/qualitativo } \\
\text { exploratório }\end{array}$ & $\begin{array}{c}\text { EMP / EF } \\
\text { (7ㅇao } \\
\text { 9ano) }\end{array}$ \\
\hline $\begin{array}{l}\text { Bonde do cine: uma } \\
\text { experiência Inter } \\
\text { setorial de educação } \\
\text { popular em saúde }{ }^{15}\end{array}$ & $\begin{array}{c}\text { Ferrugem RD, Funk CS, } \\
\text { Souza RD, Machry DS, } \\
\text { Souza CC/ } \\
\text { 2015/assistente social, } \\
\text { odontóloga, psicóloga e } \\
\text { enfermeira/ RS }\end{array}$ & $\begin{array}{c}\text { Rev APS/relato de } \\
\text { experiência-qualitativa }\end{array}$ & $E P / E M$ \\
\hline $\begin{array}{c}\text { O convívio do } \\
\text { adolescente com } \\
\text { HIV/aids e o } \\
\text { autocuidado: estudo } \\
\text { descritivo }^{16}\end{array}$ & $\begin{array}{c}\text { Filho FJLS, Gubert FA, } \\
\text { Pinheiro PNC, Martins } \\
\text { AKL, Vieira NFC, Nóbrega } \\
\text { MFB/enfermeiros/2013 / } \\
\text { CE. }\end{array}$ & $\begin{array}{c}\text { OBJN/relato de } \\
\text { experiência - qualitativa }\end{array}$ & $\mathrm{AH}$ \\
\hline $\begin{array}{c}\text { Impacto de um } \\
\text { programa de educação } \\
\text { nutricional em } \\
\text { adolescentes } \\
\text { estudantes da rede } \\
\text { pública }{ }^{17}\end{array}$ & $\begin{array}{c}\text { Horta PM, Ferreira AD, } \\
\text { Santos } \\
\text { LC/nutricionista/2012/MG }\end{array}$ & $\begin{array}{c}\text { Rev APS/ intervenção - } \\
\text { qualitativa }\end{array}$ & $\mathrm{EP} / \mathrm{EF}$ \\
\hline
\end{tabular}


(Continuação)

\begin{tabular}{|c|c|c|c|}
\hline Título & $\begin{array}{l}\text { Autor/ano/equipe } \\
\text { pesquisadora/local }\end{array}$ & $\begin{array}{l}\text { Periódico/tipo de } \\
\text { estudo }\end{array}$ & $\begin{array}{l}\text { Local } \\
\text { da } \\
\text { interv. }\end{array}$ \\
\hline $\begin{array}{c}\text { Dinâmicas de } \\
\text { criatividade e } \\
\text { sensibilidade na } \\
\text { abordagem de álcool e } \\
\text { fumo com } \\
\text { adolescentes }^{18} \\
\end{array}$ & $\begin{array}{c}\text { Lopes GT, Priscila CB, } \\
\text { Ingryd CVF et } \\
\text { al./2012/enfermeiro, } \\
\text { publicitário e acadêmicos } \\
\text { de Enfermagem/RJ }\end{array}$ & $\begin{array}{l}\text { Rev Enferm/qualitativo - } \\
\text { relato de experiência }\end{array}$ & $\begin{array}{l}\text { EP / EF } \\
\text { (quatro } \\
\text { turmas } \\
\text { do 6o } \\
\text { ano) }\end{array}$ \\
\hline $\begin{array}{c}\text { Expressão livre de } \\
\text { jovens por meio do } \\
\text { fanzine: recurso para a } \\
\text { terapia ocupacional } \\
\text { sociall }\end{array}$ & $\begin{array}{l}\text { Lopes RE, Borba PLO, } \\
\text { Monzeli GA./terapeuta } \\
\text { ocupacional/2013/ SP }\end{array}$ & $\begin{array}{l}\text { Saúde Soc/qualitativo - } \\
\text { pesquisa-intervenção. }\end{array}$ & $P$ \\
\hline $\begin{array}{l}\text { Promoção da saúde do } \\
\text { adolescente em } \\
\text { ambiente escolar }\end{array}$ & $\begin{array}{c}\text { Martins AS, Horta NC, } \\
\text { Castro MCG/enfermeiros } \\
\text { e assistente social/ } \\
\text { 2013/MG }\end{array}$ & $\begin{array}{c}\text { Rev APS/intervenção - } \\
\text { qualitativa }\end{array}$ & $\begin{array}{l}\text { EMp } \\
\text { ( } 5 \underline{a} \\
\text { série) }\end{array}$ \\
\hline $\begin{array}{l}\text { Grupo de educação em } \\
\text { saúde com } \\
\text { adolescentes de uma } \\
\text { comunidade adscrita a } \\
\text { uma USF: uma } \\
\text { experiência de } \\
\text { aprendizado no âmbito } \\
\text { do programa de } \\
\text { educação pelo } \\
\text { trabalho }\end{array}$ & $\begin{array}{c}\text { Melo } \\
\text { GC/enfermeiros/2014 / } \\
\text { AL }\end{array}$ & $\begin{array}{c}\text { Rev APS/relato de } \\
\text { experiência - qualitativa. }\end{array}$ & $\begin{array}{c}\mathrm{EP} / \mathrm{EF} / \\
\mathrm{EM}\end{array}$ \\
\hline $\begin{array}{c}\text { Vivências do } \\
\text { adolescente com } \\
\text { HIV/aids }\end{array}$ & $\begin{array}{c}\text { Motta MGC, Pedro ENR, } \\
\text { Paula CC et al./médico, } \\
\text { psicólogo e } \\
\text { enfermeiro/2014/RS }\end{array}$ & $\begin{array}{l}\text { Reme/qualitativa- } \\
\text { relato de experiência }\end{array}$ & SE \\
\hline $\begin{array}{c}\text { Programa de } \\
\text { habilidades } \\
\text { interpessoal e direito } \\
\text { sexual e reprodutivo } \\
\text { para adolescentes: um } \\
\text { relato de experiência }{ }^{23}\end{array}$ & $\begin{array}{l}\text { Murta SG, Danilo CR, } \\
\text { Isabela OR et } \\
\text { al./Psicólogos/2012/ GO }\end{array}$ & $\begin{array}{l}\text { Psico - USF/ Relato de } \\
\text { experiência - qualitativa. }\end{array}$ & $\mathrm{EP} / \mathrm{EF}$ \\
\hline $\begin{array}{c}\text { Educação sexual de } \\
\text { adolescentes na } \\
\text { perspectiva freireana } \\
\text { através dos círculos de } \\
\text { cultura }^{24}\end{array}$ & $\begin{array}{c}\text { Nau AL, Boca SS, Schülter } \\
\text { BHIT, Maria GM, Castillo } \\
\text { L/médico, enfermeiro, } \\
\text { acadêmico de } \\
\text { Medicina/2013/SC }\end{array}$ & $\begin{array}{l}\text { Revrene/Qualitativo- } \\
\text { pesquisa-intervenção }\end{array}$ & $\begin{array}{l}\text { EF (7a e e } \\
\text { 8a série) }\end{array}$ \\
\hline $\begin{array}{l}\text { Violência sob o olhar de } \\
\text { adolescentes: } \\
\text { intervenção educativa } \\
\text { com círculos de } \\
\text { cultura }^{25}\end{array}$ & $\begin{array}{l}\text { Brandão NW, Silva MAI, } \\
\text { Lima LS et } \\
\text { al./enfermeiros/2015/ PE }\end{array}$ & $\begin{array}{c}\text { Reben/pesquisa-ação - } \\
\text { qualitativa. }\end{array}$ & EE / EM \\
\hline
\end{tabular}




\begin{tabular}{|c|c|c|c|}
\hline & & & Conclusão) \\
\hline Título & $\begin{array}{l}\text { Autor/ano/equipe } \\
\text { pesquisadora/local }\end{array}$ & $\begin{array}{c}\text { Periódico/tipo de } \\
\text { estudo }\end{array}$ & \begin{tabular}{|c|}
$\begin{array}{c}\text { Local } \\
\text { da } \\
\text { interv. }\end{array}$ \\
\end{tabular} \\
\hline $\begin{array}{l}\text { Intervenção educativa } \\
\text { sobre violência com } \\
\text { adolescentes: } \\
\text { possibilidade para a } \\
\text { Enfermagem no } \\
\text { contexto escolar }{ }^{26}\end{array}$ & $\begin{array}{c}\text { Neto WB, Andrea RSS, } \\
\text { Antônio JAF et } \\
\text { al./enfermeiros/2014/PE }\end{array}$ & $\begin{array}{c}\text { Eean/pesquisa-ação - } \\
\text { qualitativa }\end{array}$ & EESI \\
\hline $\begin{array}{c}\text { Cotidiano de } \\
\text { adolescentes com o } \\
\text { vírus da } \\
\text { imunodeficiência } \\
\text { humana em } \\
\text { tratamento } \\
\end{array}$ & $\begin{array}{c}\text { Paula CC, Stela MMP, } \\
\text { Paulo VCA et } \\
\text { al./enfermeiros e } \\
\text { acadêmicos de } \\
\text { Enfermagem/ 2013/ RS }\end{array}$ & $\begin{array}{c}\text { Rev Enferm UFSM/relato } \\
\text { de experiência - } \\
\text { qualitativa }\end{array}$ & $\mathrm{HU}$ \\
\hline $\begin{array}{c}\text { Educação em saúde } \\
\text { com adolescentes } \\
\text { acerca do uso de álcool } \\
\text { e outras drogas }{ }^{28}\end{array}$ & $\begin{array}{c}\text { Pedrosa SC, Costa DVS, } \\
\text { Citó MCO et } \\
\text { al./2015/enfermeiro/CE }\end{array}$ & $\begin{array}{l}\text { Recom/qualitativo- } \\
\text { relato de experiência }\end{array}$ & $\begin{array}{c}\mathrm{EMp} / \\
\mathrm{EM}\end{array}$ \\
\hline $\begin{array}{c}\text { Educação em saúde } \\
\text { para adolescentes de } \\
\text { uma escola municipal: a } \\
\text { sexualidade em } \\
\text { questão } \\
\end{array}$ & $\begin{array}{c}\text { Pinto MB, Santos NCCB, } \\
\text { Albuquerque AM, } \\
\text { Ramalho MNA, Torquato } \\
\text { IMB/enfermeiros e } \\
\text { acad./2013/PB } \\
\end{array}$ & $\begin{array}{c}\text { Cienc Cuid } \\
\text { Saúde/qualitativo - } \\
\text { relato de experiência }\end{array}$ & $\mathrm{EMp} / \mathrm{EF}$ \\
\hline $\begin{array}{c}\text { Estudantes de Medicina } \\
\text { ensinam Ressuscitação } \\
\text { Cardiopulmonar a } \\
\text { Alunos do } \\
\text { Fundamental } \\
\text { Fun }^{30} \\
\end{array}$ & $\begin{array}{c}\text { Ribeiro LG, Rafael G, } \\
\text { Pedro LM, André S, } \\
\text { Antônio PF/médicos e } \\
\text { acadêmicos de } \\
\text { Medicina/2013/SP } \\
\end{array}$ & $\begin{array}{c}\text { SBC / Qualitativo - } \\
\text { pesquisa -intervenção }\end{array}$ & $\begin{array}{l}\text { EP e EPr } \\
\text { / EF }\end{array}$ \\
\hline $\begin{array}{l}\text { Efeitos das drogas } \\
\text { lícitas e ilícitas na } \\
\text { percepção de } \\
\text { adolescentes: uma } \\
\text { abordagem de } \\
\text { Enfermagem }{ }^{31}\end{array}$ & $\begin{array}{c}\text { Silveira HS, Ferreira VS, } \\
\text { Zeitoune Artigo de } \\
\text { Pesquisa RCG, Domingos } \\
\text { AM/enfermeiros/2013/RJ }\end{array}$ & $\begin{array}{c}\text { Rev } \\
\text { Enferm/quantiqualitativo }\end{array}$ & CCRJ \\
\hline $\begin{array}{l}\text { Educação em saúde } \\
\text { com adolescentes: } \\
\text { análise da aquisição de } \\
\text { conhecimentos sobre } \\
\text { temas de saúde } \\
2\end{array}$ & $\begin{array}{c}\text { Viero VSF, Farias JM, } \\
\text { Ferraz F, Simões PW, } \\
\text { Martins JA, Ceretta } \\
\text { LB/enfermeiros./2015 /RS }\end{array}$ & EEAN/ qualitativa-ação & $\begin{array}{c}\mathrm{EE} / \mathrm{EF} \text { e } \\
\mathrm{EM}\end{array}$ \\
\hline $\begin{array}{c}\text { Conhecimentos sobre } \\
\text { tabagismo em } \\
\text { escolares }^{33}\end{array}$ & $\begin{array}{c}\text { Vitta AD, Daniela TS, } \\
\text { Fabiana CFV et } \\
\text { al./2013/fisioterapeuta, } \\
\text { terapeuta Ocupacional, } \\
\text { enfermeiro, } \\
\text { odontólogo/SP }\end{array}$ & $\begin{array}{c}\text { JHGD/qualitativo- relato } \\
\text { de experiência }\end{array}$ & $\begin{array}{l}\text { EF (cinco } \\
\text { quintas } \\
\text { séries) }\end{array}$ \\
\hline $\begin{array}{l}\text { Vulnerabilidade e } \\
\text { autonomia na pesquisa } \\
\text { com adolescentes } \\
\text { privados de liberdade }{ }^{34}\end{array}$ & $\begin{array}{c}\text { Zappe } \\
\text { JG/psicólogo/2013/RS }\end{array}$ & $\begin{array}{l}\text { Psicologia: ciência e } \\
\text { profissão/relato de } \\
\text { Experiência }\end{array}$ & CCMS \\
\hline
\end{tabular}


Legenda: AH - Ambulatório de um hospital; CCMS - Centro de cumprimento de medidas socioeducativas; CCRJ - Comunidade na cidade do Rio de Janeiro; E - Escola; EE - Escola estadual; EESI - Escola estadual semi-integral; EF - Ensino fundamental; EJA - Educação de Jovens e Adultos; EM - Ensino médio; EMp Escola municipal; EP - Escola pública; EPr - Escola privada; HU - Hospital universitário; P - Periferia; SE Serviços especializados

\section{Fonte: elaborado pelos autores}

Como resultados da utilização dos descritores "adolescente", "educação" e "saúde" e do booleano "and", encontraram-se 40.831 publicações. Restringindo para títulos em português, foram obtidos 3.014 estudos. Limitando-se para texto completo disponível gratuitamente, 1.518 foram selecionados - destes 1.294 eram artigos e 468 publicados nos últimos cinco anos (2012, 2013, 2014, 2015 e 2016).

Com a leitura de títulos e resumos, excluíram-se 81 artigos por estarem repetidos; 38, por tratarem de revisão de literatura; e 346, por não responderem à questão-problema. Dessa forma, restaram 26 artigos que foram lidos na íntegra e analisados na presente pesquisa.

Como resultado, quanto ao ano de publicação, evidenciou-se que, dos últimos cinco anos, 2013 foi o ano em que mais houve publicações referentes ao tema pesquisado, com 13 artigos, seguido de 2012 (com seis publicações), 2014 e 2015, ambos com quatro publicações, e 2016 sem publicações.

Em relação aos profissionais envolvidos com a publicação dos estudos, 19 artigos contaram com a presença de enfermeiros, seis com psicólogos, cinco com acadêmicos de Enfermagem e três com médicos. Assistentes sociais, terapeutas ocupacionais, odontólogos e acadêmicos de Medicina estiveram presentes em dois estudos cada; e fisioterapeutas, publicitários, fonoaudiólogos e nutricionistas participaram de apenas um estudo.

Com relação ao local de realização da pesquisa, a região Nordeste destacou-se com dez publicações (seis do Ceará, dois de Pernambuco, um da Paraíba outro de Alagoas); seguida da região Sudeste, com oito estudos (cinco do Rio Grande do Sul, dois de Minas Gerais e um de Goiás); e da região Sul, com sete (quatro de São Paulo, dois do Rio de Janeiro e um de Santa Catarina). Na região Centro-Oeste, constatou-se um estudo no estado do Paraná, e não foram encontradas publicações de estudos da região Norte.

As publicações originam-se de diversas revistas. A Revista APS - Atenção Primária à Saúde foi a que obteve o maior número de publicações, com o total de quatro 
artigos. A Revista de Enfermagem da Escola Anna Nery (Eeam) integrou três estudos. A Revista Mineira de Enfermagem (Reme), dois; e a Revista de Enfermagem da Universidade Estadual do Rio de Janeiro (Uerj), dois, bem como a Ciência, Cuidado e Saúde, da Universidade Estadual de Maringá (UEM). As demais revistas tiveram uma publicação, Journal of Human Growthand Development, Revista de Enfermagem do Centro-Oeste Mineiro (Recom), Psicologia em Estudo, Revista da Rede de Enfermagem do Nordeste (Revrene), Sociedade Brasileira de Cardiologia (SBC), Saúde e Sociedade, Revista de Enfermagem da Universidade Federal de Santa Maria, Audiology Communication Research: ACR, Revista Brasileira de Enfermagem (Reben), Psicologia: Ciência e Profissão, Estudos e Pesquisas em Psicologia, Psicologia -Universidade São Francisco (USF); e Online Brazilian Journal of Nursing (OBJN).

Em relação aos tipos dos estudos, 24 artigos utilizaram a abordagem qualitativa. A metodologia mista (qualitativo-quantitativa) esteve presente em duas pesquisas. A pesquisa-intervenção foi apresentada em 21 artigos; e a pesquisa-ação, em cinco.

Dos 26 artigos analisados, identificaram-se diversos tipos de estratégias usadas para a aplicação de intervenções com os adolescentes. As oficinas educativas foram relatadas em 12 pesquisas. Já os questionários foram usados em seis estudos, e citaramse o método freiriano e o círculo de cultura cinco vezes cada. O diário de campo foi um método usado em três destes. Foram citados em dois artigos: "Jogos educativos, Método Criativo Sensível (MCS), discussão grupal"; e "Dinâmicas de Criatividade e Sensibilidade (DCS), Vídeos ou Filmes e Entrevistas". Estiveram presentes em apenas um estudo as aulas expositivas, o grupo focal, a técnica do "ver e praticar", o método fanzine, as oficinas de acordo com Monteiro e Rabelo (2005) e a metodologia interacionista.

Na pesquisa, foi possível identificar que dois artigos não informaram o período de duração das estratégias. Dois artigos trabalharam suas ações em apenas dois encontros, três estudos tiveram três encontros; um mês, dois meses e cinco meses foram o tempo citado em dois artigos, cada. Em apenas um artigo, citou-se individualmente a duração de quatro meses. Sete, 12, 15 encontros, 15 semanas e dois anos. Um estudo durou um ano, e quatro duraram um semestre.

Quanto às temáticas abordadas nas ações: 16 eram sobre saúde sexual e reprodutiva, nove acerca de drogas e uma sobre tabagismo. Autonomia e 
voluntariedade, higiene, tratamento antirretroviral e saúde bucal foram assuntos de intervenção em dois artigos cada. Preconceito, autocuidado, ressuscitação cardiopulmonar, saúde auditiva e educação alimentar e nutricional foram trabalhados em apenas um estudo cada.

As escolas mostraram-se como os cenários de maior escolha pelos artigos, em 21 publicações. Dessas instituições de ensino, 13 eram de nível fundamental, sete do ensino médio e um Educação de Jovens e Adultos (EJA). Dois artigos citaram os hospitais como escolha para a aplicação das estratégias. Houve também locais citados em apenas um artigo, como periferia, comunidade, centros especializados e unidade regional para cumprimento de medida socioeducativa.

De forma geral, as ações foram consideradas positivas pelos autores dos estudos. Apenas em um evidenciou-se a necessidade de melhoria nas estratégias. Dos artigos, 96\% afirmaram aumento do conhecimento, inclusive de forma crítico-reflexiva quanto às diversas temáticas abordadas. Acrescenta-se que sete pesquisas afirmaram o incremento do número de participantes das ações em seu decorrer, como um feedback positivo para suas realizações.

Ensinar não se trata apenas de transmitir os conhecimentos e conteúdos, nem formar. É uma ação pela qual um sujeito criador dá forma, alma, a um corpo indeciso e acomodado. Não há docência sem discência. As duas explicam-se. E seus sujeitos, apesar das diferenças, não se reduzem à condição de objeto, um do outro. Quem ensina aprende ao ensinar e quem aprende ensina ao aprender. ${ }^{35}$

Avaliaram-se as estratégias de diferentes formas entre os artigos pesquisados. Alguns utilizaram mais de uma forma de avaliação. Oito artigos citaram que houve uma afirmação positiva por parte dos adolescentes relatando a assimilação dos conhecimentos aplicados. Sete pesquisas avaliaram suas estratégias pelo aumento dos diálogos e debates críticos com os profissionais envolvidos. Materiais artísticos e textos redigidos pelos participantes foram usados para avaliar a apropriação do conhecimento em intervenções aplicadas em seis artigos. Cinco estudos aplicaram questionários como avaliação.

Mencionaram-se avaliações coletivas com retorno positivo em três artigos. Foram empregadas análises estatísticas em três artigos. Em três artigos, observou-se o uso de metodologias de análise de discurso obtido em grupo para afirmar a eficácia da 
intervenção realizada. A construção de paródias foi usada para avaliar o conhecimento após intervenções em dois artigos. Um artigo utilizou a atividade lúdica associada a uma entrevista com questões abertas. Evidencia-se a falta de detalhamento da forma como a avaliação da estratégia foi realizada pelas publicações, o que limitou algumas conclusões.

Inicia-se a discussão com a evidência do ano de publicação dos artigos. Entre os cinco anos utilizados para a pesquisa, os anos anteriores ganharam destaque em publicações, mostrando uma queda de publicações atualmente quanto ao tema. Como a data de coleta de dados foi em meados de 2016, pode-se justificar a não constatação de publicações.

Em 2007, iniciou-se um programa pelo Ministério da Educação, vinculado com o Ministério da Saúde, denominado Programa Saúde na Escola (PSE), que priorizava o desenvolvimento intelectual dos adolescentes através da educação em saúde nas escolas, abordando temas relevantes à fase da puberdade. Em 2013, cerca de 80\% dos adolescentes brasileiros que frequentavam escolas receberam orientações de prevenção e promoção à saúde, de acordo com pesquisas nacionais. ${ }^{36}$

É importante destacar o valor da formação de vínculos para que haja a continuidade dos programas e ações de incentivos à educação em saúde com os adolescentes. Quanto aos profissionais envolvidos com as intervenções realizadas com os adolescentes, apesar de existir uma grande diversidade de profissionais, o que mostra o interesse quanto ao assunto, $73 \%$ dos profissionais eram enfermeiros. Vale destacar que nenhum dos descritores de busca utilizados foi da área exclusiva da Enfermagem.

A Enfermagem possui um papel educativo que contribui para a promoção da saúde e prevenção de doenças e agravos, em que o dever do "cuidado" vai além da cura de males, conquistando seu espaço nas intervenções sociais, ambientais e educativas. ${ }^{9}$ O Ministério da Saúde corrobora essa afirmação e acrescenta que as ações inter e multiprofissional devem ocorrer como uma busca constante, mas o surgimento de problemas ou impossibilidade não pode ser um empecilho. Atividades específicas podem ser feitas apenas com um profissional que tenha interesse em realizar as ações e, gradualmente, instigar outros profissionais para a realização do trabalho em equipe. Todas as classes de profissionais podem qualificar-se para o acolhimento de 
adolescentes e jovens. A equipe multiprofissional contribui para aumentar as possibilidades de atuação e resolução de problemas. ${ }^{37}$

O Programa de Saúde da Família surgiu na região Nordeste, sendo implantado em pequenos municípios, alcançando, depois de uma década, os grandes centros urbanos do país. A Estratégia de Saúde da família conquistou um espaço em todo o país, sendo adotada pelo Ministério da Saúde como referência, tornando-se uma política de Estado e um dos pilares de sustentação do Sistema Único de Saúde. Assim, é provável que a região Nordeste tenha conquistado mais estabilidade e inserção, garantindo maior número de adesão da população ao sistema. ${ }^{38}$ Por outro lado, a região Norte é a que possui um dos menores índices de educação do país, o que pode gerar maior dificuldade de acesso da população ao desenvolvimento de pesquisas ou, até mesmo, das estratégias de educação em saúde. ${ }^{39}$

A maior parte das publicações foi em revistas voltadas para a área da Atenção Básica, sendo uma área de grande atuação da Enfermagem. As revistas de Enfermagem ganharam destaque de publicações na pesquisa. Seguiram-se revistas voltadas para a Psicologia e a Medicina. As demais abordavam um contexto multidisciplinar.

A participação de revistas de Enfermagem em bases de dados comprova os avanços quantitativos e qualitativos na produção do conhecimento da área. Mesmo o crescimento do número de revistas brasileiras em outras bases confirma esse fato. Publicar e compartilhar achados, novos dados, pensamentos críticos e aprofundados, de alguma maneira, contribuem para a qualificação das práticas profissionais em saúde. ${ }^{40}$

As pesquisas do tipo qualitativas compuseram $89 \%$ dos textos. Delas, $11 \%$ utilizaram uma metodologia mista, qualiquantitativa. Dos artigos, $81 \%$ empregaram a abordagem do tipo relato de experiência e 19\% optaram pela pesquisa-ação.

A pesquisa qualitativa não se preocupa com estatísticas, mas, sim, com o aprofundamento da concepção do grupo abordado. Está ligada ao diagnóstico dos sentidos que vão ganhando de modo crescente a integralização aos aprendizados. 0 qualitativo refere-se, então, à probabilidade de restaurar os abalos da comunidade, percebido nos conflitos, nas desavenças, frente às influências, para um futuro indeterminado. ${ }^{41}$

A pesquisa-intervenção não busca a transformação imediata da ação instituída, 
pois a modificação decorre da produção de uma analogia entre teoria e prática, bem como entre sujeito e objeto. Na pesquisa-intervenção, a relação pesquisador/objeto pesquisado é eficaz e gera os próprios caminhos da pesquisa, sendo um resultado da equipe envolvida. ${ }^{42}$

Em 46\% dos artigos, a oficina educativa esteve presente como estratégia de educação em saúde com os adolescentes. Destaca-se que alguns autores não foram específicos sobre a metodologia utilizada na ação, porém possuíam características semelhantes à de uma oficina. Dessa forma, a porcentagem de uso de oficinas pode ser maior que a apresentada.

As oficinas educativas possibilitam a quebra do tabu existente no contato direto entre o profissional e o sujeito, sendo uma estratégia que permite a abertura livre do individuo e do coletivo quanto às necessidades, perspectivas e situações de vida que influenciam a saúde. Tal fato permite a formação da educação coletiva e a unificação da reflexão com a ação. ${ }^{10}$

Ainda se destacaram os questionários; o método freiriano, com o círculo de cultura; e o diário de campo. Outras formas também foram citadas em menor frequência, como uso de vídeos ou filmes, aulas expositivas e jogos educativos, entre outros.

O método de Paulo Freire valoriza as raízes culturais do indivíduo, que se mostram no círculo de cultura, um termo nomeado pelo autor, constituído por um ambiente oportunizador da troca de aprendizagem e de conhecimento referente a temas de interesse do próprio grupo ou da comunidade. O diário de campo é utilizado para o registro das atividades dos círculos de cultura com o objetivo de aperfeiçoar a qualidade e a fidelidade dos dados coletados. ${ }^{43}$

As ações de educação em saúde duraram, em média, seis encontros. Uma ação teve o período de dois anos. Três artigos relatam uma duração de seis meses de ações. Um artigo descreve o tempo de um mês. Dessa maneira, prevaleceram as mais extensas que assinalavam uma necessidade de organização dos profissionais para realizá-las.

Os artigos relatam a escola como espaço de intervenção em $81 \%$ dos pesquisados; $15 \%$ ocorreram em periferia, comunidade, centros especializados e unidade regional para o cumprimento de medida socioeducativa. Apenas $7 \%$ das pesquisas ocorreram na área hospitalar, percebendo-se maior dificuldade ou resistência 
à realização de atividades de educação em saúde nesse âmbito.

Desde o surgimento dos primeiros hospitais, vem-se tentando desconstruir a cultura apenas curativa dos hospitais. A Enfermagem ganha destaque quanto às lutas de melhorias de assistência ao paciente, em que o hospital deixa de ser apenas uma instituição que reestabelece a saúde para desempenhar uma função mais abrangente na recuperação, na manutenção e na prevenção da doença. ${ }^{44}$

Salienta-se que $61 \%$ dos artigos abordavam educação sexual e reprodutiva como tema de suas ações. Drogas foram abordadas em $38 \%$ dos estudos. Outros temas foram relatados em uma menor escala: Saúde Bucal, autocuidado, preconceito, entre outros.

A saúde sexual e reprodutiva vem ganhando destaque em meios de referência. A Organização das Nações Unidas (ONU) ressaltou na Conferência do Milênio, no ano de 2000 , oito objetivos de desenvolvimento. A saúde sexual e reprodutiva estava ligada diretamente em quatro destes objetivos. No Brasil, o Pacto pela Saúde, estabelecido em 2006 pelos gestores do Sistema Único de Saúde (SUS), também engloba a saúde sexual e a saúde reprodutiva entre suas prioridades. ${ }^{45}$

Indicadores apontam que o abuso de drogas vem assumindo uma dimensão preocupante, acarretando estragos à população, sobretudo aos jovens e adolescentes. A insuficiência de recursos financeiros que garantam um planejamento de educação, prevenção e tratamento de forma adequada, a baixa efetividade dos programas e a baixa produção do conhecimento refletem uma baixa prioridade política atribuída ao tema. ${ }^{46}$

Em média, 69\% dos artigos não utilizaram base metodológica para avaliar a efetividade das intervenções. Evidenciaram-se as limitações das publicações quanto às conclusões e avaliações das estratégias utilizadas de modo superficial.

Para se avaliar a efetividade de uma atividade, o aprendizado e o aproveitamento, é importante a utilização de técnicas metodológicas que contribuam para o desenvolvimento e o aperfeiçoamento da educação em saúde. As metodologias propiciam melhor abordagem e fidedignidade quanto aos resultados, contribuindo para pesquisadores e profissionais que poderão utilizar a estratégia avaliada. A avaliação das metodologias contribui de forma efetiva na qualificação das práticas de atenção à saúde, na aprendizagem, na mudança e na reconstrução do cotidiano, na sensibilização dos profissionais e no planejamento e organização das atividades do serviço de forma 
eficiente e confiável. ${ }^{47}$

\section{CONCLUSÃO}

A educação em saúde com adolescentes é uma área muito desafiadora e de grande relevância para o desenvolvimento e a contribuição da sociedade. A adolescência mostra-se um período de formação da identidade social, que requer uma atenção especial por parte dos profissionais e familiares. A escolha de metodologias para a construção de ações educacionais é de extrema importância.

Após a análise dos dados, foi possível evidenciar as estratégias de educação em saúde realizadas com adolescentes, embora sendo avaliadas de formas superficiais e pouco eficazes. Ressalta-se que as metodologias participativas são de grande contribuição para o desenvolvimento educacional, reflexivo e de diálogo entre os profissionais e os adolescentes. Identificou-se a desvalorização de utilização de metodologias embasadas cientificamente, para a melhor avaliação das ações educativas.

Observou-se que a Enfermagem é a área que vem se destacando nas publicações, nas realizações de intervenções e nas ações educativas. Salienta-se a inserção de uma grande variedade de profissionais envolvidos com pesquisas nesta área de conhecimento.

Percebeu-se uma grande variedade de políticas públicas voltada para a saúde do adolescente. No entanto, são poucas as efetivadas e priorizadas para a construção e a formação social do adolescente, em que se predominam ambientes escolares para a iniciativa de estratégias educacionais.

Conclui-se que a pesquisa é de grande contribuição e significado social, por garantir um melhor desenvolvimento crítico reflexivo do profissional quanto à formação e à construção de sua identidade. Portanto, são necessárias mais pesquisas voltadas para a educação em saúde com os adolescentes.

Reforça-se a importância da pesquisa na área profissional. Isso porque ela nos garante a busca de novas metodologias e avaliações estratégicas, reportando-nos para o valor das práticas baseadas em evidências, para uma melhor aplicabilidade de forma dinâmica, afável e pessoal. 


\section{REFERÊNCIAS}

1. Brasil. Secretaria de Atenção à Saúde. Departamento de Ações Programáticas Estratégicas. Proteger e cuidar da saúde de adolescentes na atenção básica. 2. ed. Brasília: Ministério da Saúde, 2018.

2. Ferreira MA. A educação em saúde na adolescência: grupos de discussão como estratégia de pesquisa e cuidado-educação. Texto Contexto Enferm. 2006; 15(2):215-11.

3. Ressel LB, Sehne GD, Junge CF, Hoffmann IC, LanderdahI MC. Representações culturais de saúde, doença e vulnerabilidade sob a ótica de mulheres adolescentes. Esc Anna Nery Ver Enferm. 2009; 13(3):552-7.

4. Ferreira CPS, Marques JF, Rozendo CA, Ferreira CB, Pinto LMTR, Ferreira AS. Estratégias pedagógicas para educação em saúde com adolescentes: uma revisão integrativa. J Res Fundam Care Online. 2016. 8(2):4197-4211.

5. Santos JS, Andrade RD, Mello DFD, Maia MAC. Educação em saúde na adolescência: contribuições da Estratégia Saúde da Família. Rev Soc Bras Enferm Ped. 2014. 14(1):20-6.

6. Trindade LDL, Ferraz L, Zanatta EA, Bordignon M, Mai S. Vulnerabilidade na adolescência: a ótica dos enfermeiros da saúde da família. 17o Senpe. 2013; 174-6.

7. Siqueira FBS, Ressel LB, Sanfelice C, Pugin TT, Barreto CN. Adolescer, crescer e viver: o trabalho de alunos de enfermagem com adolescentes. Anais 25a JAl; 2010.

8. Mendes KDS, Silveira RCCP, Galvão C. Revisão integrativa: método de pesquisa para a incorporação de evidências na saúde e na Enfermagem. Texto Contexto Enferm. 2008. 17(4):758-64.

9. Coelho MMF, Raimundo AMT, Karla CLM, Cabral RL, Almeida LKG, Queiroz MVO. Educação em saúde com adolescentes: compartilhando Vivências e reflexões. Ciênc Cuid Saúde. 2012. 11(2):390-395.

10. Lacerda ABM, Soares VMN, Goncalves CGO, Lopes FC, Testoni R. Oficinas educativas como estratégia de promoção da saúde auditiva do adolescente: estudo exploratório. ACR. 2013. 18(2):85-92.

11. Barros JPP, Rodrigues VF. Sentidos sobre "sexualidade" e "drogas" entre adolescentes no contexto escolar. Estudos Pesq Psicol. 2013. 13(1):63-85.

12. Bezerra EP, Sousa LBM, Alves DS. Intervenção educativa utilizando a atividade de vida respiração com adolescentes. Escola Anna Nery Rev Enferm. 2014. 8(2): 20914.

13. Bortolozzi ACM, Eidt NM, Terra BM, Maia GL. Educação sexual na escola a partir da Psicologia histórico-cultural. Psicologia em Estudo. 2012. 17(1):151-6.

14. Ferreira AGN, Silva KL, Sousa PRM, Gubert FA, Vieira NFC, Pinheiro PNC. Cultura masculina e religiosidade na prevenção das DSTs/HIV /aids em adolescentes. Rev Min Enferm. 2012; 16(4): 572-578. 
15. Ferrugem RD, Funk CS, Souza RD, Machry DS, Souza CC. Bonde do cine: uma experiência Inter setorial de educação popular em saúde. Rev APS. 2015; 18(4): 507-11.

16. Filho FJLS, Gubert FA, Pinheiro PNC, Martins AKL, Vieira NFC, Nóbrega MFB. O convívio do adolescente com HIV/AIDS e o autocuidado: estudo descritivo. Braz J Nurs. 2013; 12(1):89-105.

17. Horta PM, Ferreira AD, Santos LC. Impacto de um programa de educação nutricional em adolescentes estudantes da rede pública. Rev APS. 2012; 15(2):18591.

18. Lopes GT, Priscila CB, Ingryd CVF, Bernardes MM, Casanova EG, Pinheiro APL. Dinâmicas de criatividade e sensibilidade na abordagem de álcool e fumo com adolescentes. Rev Enferm. 2012; 20(1):33-8.

19. Lopes RE, Borba PLO, Monzeli GA. Expressão livre de jovens por meio do fanzine: recurso para a terapia ocupacional social. Saúde Soc. 2013; 22(3):937-48.

20. Martins AS, Horta NC, Castro MCG. Promoção da saúde do adolescente em ambiente escolar. Rev APS. 2013; 16(1):112-6.

21. Melo GC. Grupo de educação em saúde com adolescentes de uma comunidade adscrito a uma unidade de saúde da família: uma experiência de aprendizado no âmbito do programa de educação pelo trabalho. Rev APS. 2014; 17(2):268-72.

22. Motta MGC, Pedro ENR, Paula CC, Coelho DF, Ribeiro AC, Greff AP et al. Vivências do adolescente com HIV/aids. Rev Min Enfer. 2014; 18(1):181-7.

23. Murta SG, Ribeiro DC, Rosa IO, Menezes JCL, Ribeiro MRS, Borges OS et al. Programa de habilidades interpessoal e direito sexual e reprodutivo para adolescentes: um relato de experiência. Psico - USF. 2012; 17(1):21-32.

24. Nau AL, Boca SS, Schülter BHIT, Maria GM, Castillo L. Educação sexual de adolescentes na perspectiva freireana através dos círculos de cultura. Rev Rede Enferm Nordeste. 2013; 14(5): 886-93.

25. Brandão NW, Silva MAI, Aquino JM, Lima LS, Monteiro EMLM. Violência sob o olhar de adolescentes: intervenção educativa com círculos de cultura. Rev Bras Enferm. 2015; 68(4): 617-25.

26. Neto WB, Silva ARS, Filho AJA, Lima LS, Aquino JM, Monteiro EMLM. Intervenção educativa sobre violência com adolescentes: possibilidade para a Enfermagem no contexto escolar. Escola Anna Nery RevEnferm. 2014; 18(2): 195-201.

27. Paula CC, Padoin SMM, Albuquerque PVC, Bubaduéet RM, Silva CB, Brum CN. Cotidiano de adolescentes com o vírus da imunodeficiência humana em tratamento. Rev Enferm UFSM. 2013; 3(3): 500-8.

28. Pedrosa SC, Costa DVS, Citó MCO, Luna IT, Pinheiro PNC. Educação em saúde com adolescentes acerca do uso de álcool e outras drogas. Rev Enferm Cent O Min. 2015; 5(1):1535-41.

29. Pinto MB, Santos NCCB, Albuquerque AM, Ramalho MNA, Torquato IMB. Educação em saúde para adolescentes de uma escola municipal: a sexualidade em questão. Ciênc Cuid Saúde. 2013; (3):587-592. 
30. Ribeiro LG, Germano R, Menezes PL, Schmidt A, Filho AP. Estudantes de Medicina ensinam Ressuscitação Cardiopulmonar a Alunos do Fundamental. Soc Bras Cardiol. 2013; 101(4):328-35.

31. Silveira HS, Ferreira VS, Zeitoune Artigo de Pesquisa RCG, Domingos AM. Efeitos das drogas lícitas e ilícitas na percepção de adolescentes: uma abordagem de Enfermagem. Rev Enferm. 2013; 21(2):748-756.

32. Viero VSF, Farias JM, Ferraz F, Simões PW, Martins JA, Ceretta LB. Educação em saúde com adolescentes: análise da aquisição de conhecimentos sobre temas de saúde. Escola Anna Nery Rev Enferm. 2015; 19(3): 484-90.

33. Vitta AD, Silva DT, Vitta FCF, Conti MHS, Vieira LM, Marta SN et al. Conhecimentos sobre tabagismo em escolares. J Human Growth Developm. 2013; 23(1): 18-23.

34. Zappe JG. Vulnerabilidade e autonomia na pesquisa com adolescentes privados de liberdade. Psicologia Ciênc Prof. 2013; 33(1):234-47.

35. Freire, P. Pedagogia da autonomia: saberes necessários à prática educativa. 43. ed. São Paulo: Paz e Terra, 2011.

36. Brasil. Secretaria de Atenção à Saúde. Departamento de Atenção Básica. Saúde sexual e saúde reprodutiva. Brasília: Ministério da Saúde, 2013.

37. Brasil. Secretaria de Atenção à Saúde. Saúde integral dos adolescentes e jovens: orientações para a organização de serviços de saúde/Ministério da Saúde, Secretaria de Atenção à Saúde. Brasília: Ministério da Saúde, 2005.

38. Brasiç. Secretaria de Atenção à Saúde. Departamento de Atenção Básica. Memórias da saúde da família no Brasil / Ministério da Saúde, Secretaria de Atenção à Saúde, Departamento de Atenção Básica. Brasília: Ministério da Saúde, 2010. [Acesso em 13 set. 2016]. Disponível em: http://bvsms.saude.gov.br/bvs/publicacoes/memorias_saude_familia_brasil.pdf.

39. Portal Terra. Região Norte pede financiamento diferenciado para educação.

Agencia Brasil, 2015. [citado 2016 set 12]. Disponível em: https://noticias.terra.com.br/educacao/educacao-regiao-norte-pedefinanciamento-diferenciado-para-omec,7eaf8e46262426a5992f6c959ee46096jtx2RCRD.html.

40. Turato ER. Métodos qualitativos e quantitativos na área da saúde: definições, diferenças e seus objetivos de pesquisa. Rev Saúde Públ. 2005. 39(3):507-14.

41. Universidade Federal do Rio Grande do Sul. Curso de graduação Tecnológica Planejamento e Gestão para o Desenvolvimento Rural da Sead/UFRGS. Porto Alegre: UFRGS, 2009. [citado 2016 set 12]. Disponível em: http://www.ufrgs.br/cursopgdr/downloadsSerie/derad005.pdf.

42. Rocha ML, Aguiar KF. Pesquisa-Intervenção e a Produção de Novas Análises. Psicologia ciência e profissão. 2003. 23(4):64-73. [Acesso em: 11 set. 2016]. Disponível em: http://www.scielo.br/pdf/pcp/v23n4/v23n4a10.pdf.

43. Heidemann IBS, Boehs AE, Wosny AM, Stulp K. Incorporação teórico-conceitual e metodológica do educador Paulo Freire na pesquisa. Rev Bras Enferm. 2010; 
63(3):416-20. [citado 2016 set 13]. Disponível em: <http://dx.doi.org/10.1590/S0034-71672010000300011>.

44. Oliveira M, Oliveira MLF. Educação para saúde em unidade hospitalar: um espaço profissional do enfermeiro. Ciênc, Cuid Saúde. 2003; 2(2):131-8.

45. Brasil. Brasília: Ministério da Saúde, 2013. [2016 set 13]. Disponível em: http://www.brasil.gov.br/saude/2013/06/jovens-recebem-mais-atencao-quanto-aeducacao-sexual.

46. Raupp L, Sapiro CM. Adolescência, drogadição e políticas públicas: recortes no contemporâneo. Estudos Psicol. 2009; 26(4): 445-54. [citado 2016 set 14]. Disponível em: http://www.scielo.br/pdf/estpsi/v26n4/05.pdf.

47. Brasil. Secretaria de Atenção à Saúde. Departamento de Ações Programáticas e Estratégicas. Metodologias para o cuidado de crianças, adolescentes e famílias em situação de violências. Brasília: Ministério da Saúde, 2011.

Submissão: maio de 2017.

Aprovação: fevereiro de 2019. 